\title{
La France et les propagandes nationalistes irlandaises durant la Première Guerre mondiale
}

\section{Pierre Ranger}

\section{Q OpenEdition \\ 12 Journals}

\section{Édition électronique}

URL : http://journals.openedition.org/etudesirlandaises/1210

DOI : 10.4000/etudesirlandaises. 1210

ISSN : 2259-8863

\section{Éditeur}

Presses universitaires de Rennes

\section{Édition imprimée}

Date de publication : 30 juin 2009

Pagination : 23-36

ISBN : 978-2-7535-0935-1

ISSN : 0183-973X

\section{Référence électronique}

Pierre Ranger, «La France et les propagandes nationalistes irlandaises durant la Première Guerre mondiale », Études irlandaises [En ligne], 34.1 | 2009, mis en ligne le 30 juin 2011, consulté le 22 avril 2019. URL : http://journals.openedition.org/etudesirlandaises/1210 ; DOI : 10.4000/ etudesirlandaises. 1210

Ce document a été généré automatiquement le 22 avril 2019.

(C) Presses universitaires de Rennes 


\title{
La France et les propagandes nationalistes irlandaises durant la Première Guerre mondiale
}

\author{
Pierre Ranger
}

1 Il n'est probablement pas exagéré d'écrire que l'historiographie irlandaise de la Première Guerre mondiale a connu de très grandes évolutions ces dernières années. La publication d'un certain nombre de travaux a permis de mieux comprendre une période qui pouvait être qualifiée jusque-là d' " amnésie nationale ${ }^{1}$ ». L'identité nationale irlandaise s'est, en effet, largement développée au $\mathrm{xx}^{\mathrm{e}}$ siècle autour des figures d'un nationalisme irlandais radical, tout particulièrement celles des responsables de l'insurrection de Pâques $1916^{2}$. Patrick Pearse a été introduit au panthéon de l'histoire irlandaise après son exécution. Eamon de Valera est resté au plus proche du pouvoir de 1932, lorsqu'il devient Premier Ministre, à 1973. Qu'au moment même où quelques centaines d'hommes s'emparaient de la poste de Dublin, des milliers de soldats irlandais mourraient dans les tranchées en France sous l'uniforme britannique est, somme toute, longtemps passé inaperçu ${ }^{3}$.

2 Dans de telles circonstances, le rôle de l'Irlande dans la Première Guerre mondiale a souvent été perçu comme anecdotique par les Irlandais eux-mêmes. Il n'a certes pas été décisif, mais les dernières recherches font état d'un nombre de soldats engagés assez important au vu des handicaps évidents auquel un recrutement militaire britannique devait se confronter. Keith Jeffery dénombre 210000 soldats irlandais ayant combattu, sans compter ceux qui se sont engagés directement dans différentes parties de l'Empire britannique ou dans l'armée des États-Unis. 25000 de ces soldats ont été tués ${ }^{4}$. Outre les statistiques, les recherches les plus récentes, tels les travaux de Jérôme aan de Wiel, Ben Novick et Keith Jeffery, ont tenté de comprendre le rôle de la guerre dans l'évolution des questions intérieures irlandaises.

3 Avec le début de la guerre, la vieille et complexe division entre nationalistes irlandais constitutionnalistes modérés et séparatistes radicaux prend un tour décisif. Recherchant la mise en pratique du Home Rule que lui ont promis les Britanniques au début de la 
guerre, John Redmond, responsable du parti parlementaire irlandais, promeut une "union de cœur» et, soutenu par une large majorité de la presse irlandaise ${ }^{5}$, fait campagne pour le recrutement de soldats irlandais au sein des armées britanniques. Les nationalistes plus radicaux, très divers mais menés entre autres par le fondateur du Sinn Féin, Arthur Griffith, font front commun pour la promotion de la neutralité irlandaise. Nous définirons respectivement ces deux partis antagonistes comme les pro et les antialliés, une terminologie utilisée par Ben Novick. Mais ces termes ne reflètent pas la réalité de la situation. Un fait, notamment, rajoute une difficulté. Aucun de ces frères ennemis ne peut oublier qu'au sein des alliés, au côté de l'Angleterre, combat la France. Dans la décisive guerre de mots que se livrent les deux camps nationalistes ainsi formés, ce fait soulève maints débats, interrogations et tergiversations, largement influencés par les évolutions du contexte intérieur irlandais. Du côté anti-alliés, on peut en effet diviser la représentation donnée de la France en deux grandes phases: pré et post-Pâques 1916. Que la France soit au cœur de la propagande nationaliste irlandaise ne constitue pas, en soit, un fait nouveau ${ }^{6}$. La principale évolution se trouve dans un changement de rôle. Entre constitutionnalistes moins prompts à célébrer l'héritage français parfois violent ${ }^{7}$ du nationalisme irlandais, et des hommes plus radicaux qui par le passé en ont fait un outil de propagande, cet article cherche à comprendre la place et l'influence de la France dans un face-à-face qui, sur fond de guerre mondiale, oppose deux visions d'une Irlande indépendante.

\section{La propagande française en Irlande : enjeux et limites}

Quelques mois après le début de la guerre, le gouvernement français se montre particulièrement désireux de raviver la vieille amitié franco-irlandaise. L'objectif est triple: motiver le recrutement de soldats en Irlande, engendrer un mouvement de sympathie à l'égard des alliés dans la communauté américano-irlandaise, mais aussi aider à la stabilité de la Grande-Bretagne. Une trop grande instabilité en Irlande pourrait, en effet, conduire l'Angleterre à renforcer son contingent militaire sur l'île, et donc provoquer un affaiblissement du front ouest ${ }^{8}$. Informé de l'opinion irlandaise sur la politique française en matière de religion par le parlementaire irlandais, Timothy Power O'Connor ${ }^{9}$, le gouvernement français prend la décision, dès 1915, d'inviter à Paris une délégation d'Irlandais constitutionnalistes et catholiques. La visite a lieu du 30 avril au $1^{\text {er }}$ mai. L'objectif affiché est de renouer les sentiments amicaux des catholiques irlandais envers la France. La situation est en effet devenue préoccupante. Le clergé irlandais, tout en soutenant les alliés, semble devenir particulièrement sensible aux bonnes relations de l'Allemagne avec le Vatican ${ }^{10}$. La France, qui entretient des rapports difficiles avec le souverain pontife, et dont la réputation de pays anticlérical s'est affirmée après le vote de la Loi de Séparation de l'Église et de l'État de 1905, se doit de réagir. En effet, la baisse du recrutement en Irlande devient inquiétante ${ }^{11}$. Cette diminution est notamment le fait d'un manque de propagande en faveur du recrutement, de mauvaises décisions du cabinet Asquith qui hésite à mettre en valeur la bravoure des soldats irlandais sur le front ${ }^{12}$, et du développement économique des campagnes irlandaises pendant la guerre, qui n'invite pas les fermiers à s'engager.

5 La délégation irlandaise envoyée à Paris se compose majoritairement de membres de The Ancient Order of Hibernians ${ }^{13}$, et de son président Joseph Devlin, figure majeure du nationalisme irlandais à Belfast. Durant les trois jours que cette délégation passe dans la 
capitale française, les discours se succèdent pour promouvoir les symboles du lien francoirlandais au travers du $\mathrm{XIX}^{\mathrm{e}}$ siècle, tel le partage d'un même sang celtique. Timothy o'Connor, sur place pendant la visite, décrit par exemple la France comme « the greatest of the Celtic countries ${ }^{14}$ ", une rhétorique utilisée par John Redmond lui-même dans son manifeste sur les devoirs de l'Irlande dans la guerre :

It is a war for the defense of the sacred rights and liberties of small nations. Involved in it is the fate of France, the chief nation of that powerful Celtic race to which we belong ${ }^{15}$.

Le thème particulièrement sensible de la religion est laissé à Devlin, qui met en valeur le combat des prêtres dans les tranchées et parle d'une «France nouvelle ${ }^{16}$ ", pour mieux souligner la renaissance de la pratique religieuse dans le pays depuis le début de la guerre, ainsi que la mise en sourdine des politiques anticléricales.

Ces discours constituent donc un complet changement de ton. La France n'est plus regardée comme un pays immoral, dirigé par de violents et dangereux anticléricaux, comme avait pris l'habitude de l'affirmer le Weekly Freeman, journal catholique particulièrement proche de Redmond. Avec le début du conflit, trente années d'une rhétorique acerbe sont oubliées, et la France retrouve sa place de protectrice de la chrétienté face à la menace allemande, barbare. Le 26 décembre 1914, ce même Weekly Freeman explique :

The bombardment of Rheims cathedral has raised a wave of indignation throughout the country [...] In striking contrast to the German vandalism is the action of the French doctors, who, risking their lives, entered the Cathedral and rescued the German wounded ${ }^{17}$

Aux yeux de cette presse, la France redevient ce pays juste, humaniste, protégeant tout homme, toute nation devenue vulnérable.

\section{La campagne anti-alliés face au lien mémoriel et identitaire franco-irlandais}

Dans les deux premières années de la guerre, la réaction de plusieurs journaux anti-alliés à cette offensive pro-française est étonnamment prudente et complexe. Certes, la France, irreligieuse et amorale, apparaît une cible facile, comme le montre l'Honesty, un journal proche de l'Irish Republican Brotherhood:

The French people have practically no regard for the sanctity of marriage, and the State of France has given its sanction and recognition to immorality by legally recognizing illegitimacy ${ }^{18 .}$

10 Il est difficile de savoir à quoi fait exactement référence le journal. Peut-être à la loi Naquet de 1884 qui rétablit le divorce, ou à l'acceptation grandissante de l'« union libre » dans la société française ${ }^{19}$. Probablement cherche-t-il une parade à la rhétorique proalliée, en utilisant la représentation d'une France n'obéissant plus aux règles édictées par l'Église catholique. La dévalorisation religieuse et morale des alliés est aussi faite au 
travers d'une propagande raciale, qui dénonce l'utilisation de troupes indigènes. Griffith notamment ne se prive pas de railler dans son Nationality, «les Sénégalais et les ilotes cannibales» que l'on voudrait faire passer pour les plus grands défenseurs de la « civilisation chrétienne ${ }^{20}$ ».

$11 \mathrm{Au}$ vu de ces quelques exemples, il serait logique de conclure que l'ensemble de la presse nationaliste irlandaise anti-alliée va s'attacher à montrer au peuple d'Irlande combien la France est restée ce pays «sans Dieu ». Cependant, dans les premières années de la guerre, l'évolution n'est pas tout à fait celle-ci, et les dénonciations claires et franches de la société française sont relativement peu fréquentes ${ }^{21}$.

Cette observation peut certainement surprendre, mais elle n'en est pas moins raisonnable. Le soutien que la grande majorité des Irlandais aux sympathies nationalistes apportent dès les premiers instants de la guerre aux alliés constitue l'une des explications. Des rapports de police sur l'état de l'opinion montrent clairement que l'Allemagne est " haïe », et la France " adorée ${ }^{22}$ ». Affronter un point de vue si majoritaire en attaquant trop violemment la France et risquer de s'aliéner une partie de la population aurait été un mauvais calcul. On remarque d'ailleurs que l'article de l'Honesty est écrit dans un contexte moins favorable aux alliés où, comme on l'a vu, le recrutement est en baisse. Il faut rajouter qu'en 1914, les quelques journaux anti-alliés à faibles tirages se trouvent bien désarmés face à la puissante machinerie des grands périodiques nationaux qui appellent avec enthousiasme à soutenir l'effort de guerre français, et, surtout, qui n'hésitent pas à faire valoir le traditionnel lien franco-irlandais ${ }^{23}$.

Pour mieux comprendre cette sorte d'argument, il faut nous transporter à la fin du XIX siècle, lorsque la France joue encore un rôle important au sein du débat nationaliste irlandais ${ }^{24}$. Avant la guerre, en effet, il n'est pas rare de voir certains nationalistes plus ou moins proches de la mouvance séparatiste des fenians, prendre la défense de la politique religieuse du gouvernement français. Ainsi, au début du $\mathrm{xx}^{\mathrm{e}}$ siècle, Arthur Griffith n'hésite pas à s'opposer aux attaques de la presse catholique irlandaise contre le gouvernement français, qu'il pense instrumentalisées par les autorités britanniques pour distancier l'Irlande d'un allié ${ }^{25}$. À ces affaires religieuses se rajoute la question coloniale. Fidèles à l'adage selon lequel « les difficultés anglaises sont les opportunités irlandaises », les nationalistes irlandais dans leur grande majorité recherchent des signes de tensions franco-anglaises au cours du XIX ${ }^{e}$ siècle. Ils promeuvent dès qu'ils le peuvent le renouveau militaire français après l'humiliation de 1870 . C'est en particulier le cas de la presse proche de l'Irish Republican Brotherhood, ou en tout cas d'un certain idéal séparatiste ${ }^{26}$. Les campagnes coloniales en Afrique, à Madagascar ou au Soudan, sont l'occasion de montrer le retour au premier plan d'une armée puissante, possible rivale de celle de l'Angleterre.

La guerre des Boers leur apporte une autre raison d'espérer un soutien venu de France, ou en tout cas de le faire savoir. Si certains historiens considèrent que le gouvernement français reste plutôt neutre durant l'ensemble du conflit, d'autres travaux ont montré qu'il n'en a pas moins conscience des possibilités que peut offrir l'Irlande nationaliste. Paul Cambon, ambassadeur de France à Londres pendant la guerre des Boers, a semble-t-il pris au sérieux les risques que représente alors le mouvement nationaliste irlandais pour la sécurité de la Grande-Bretagne ${ }^{27}$. Plus généralement, les relations franco-irlandaises promues au cours $\mathrm{du} \mathrm{XIX}^{\mathrm{e}}$ siècle par les nationalistes irlandais, notamment les plus proches de l'idéal séparatiste et républicain, prennent en large partie racine dans le républicanisme de Wolfe Tone et des Irlandais Unis. Ce lien est aussi celui d'une dette que 
le pays aurait contractée avec l'intervention française pendant la révolte des Irlandais Unis.

15 La place accordée à la France dans l'histoire, la mémoire, et les politiques nationalistes irlandaises jusqu'à la fin du $\mathrm{XIX}^{\mathrm{e}}$ siècle rajoute donc une vraie complexité à la position tenue par les anti-alliés durant les deux premières années de la guerre. La preuve nous en est fournie par le Gael, lorsque le journal publie en plein milieu de la guerre une série d'articles sur les aventures de Monceau de Jones, un soldat français ayant combattu aux côtés des Irlandais Unis :

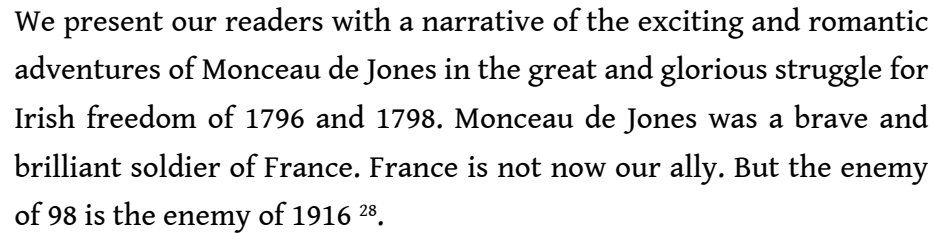

Les derniers mots tentent de dissiper l'ambiguïté de la publication de ce texte dans un tel contexte. Mais les responsables du journal ne peuvent ignorer la place de la France dans la construction d'une identité nationale irlandaise souvent tournée sur la mise en valeur d'un passé commun, des brigades irlandaises à la rébellion de 1798. Même si les relations avec la France se détériorent après 1904 et la signature de l'" Entente Cordiale ${ }^{29}$ ", pour des Irlandais nationalistes "avancés » qui tendent à se référer obsessionnellement aux faits passés, ou à ce qu'ils veulent en savoir, pour en tirer les explications, les leçons, et les justifications à leurs actes ${ }^{30}$, il est difficile de se défaire d'une telle présence historique lorsque ceux contre lesquels ils dirigent leur campagne l'utilisent dès qu'ils le peuvent.

\section{Contourner le problème français avant Pâques 1916 : une question de rhétorique}

17 Nous ne prétendons pas affirmer qu'il existe une parfaite uniformité de ton au sein des différentes publications anti-alliés, qui chacune possède une identité politique distincte. Nous ne souhaitons pas non plus nier que certains propos anti-français, notamment ceux des "Sinn Féin priests", aient été utilisés dans la presse anti-alliées ${ }^{31}$. Mais nous constatons que trois différentes approches rhétoriques ont été utilisées pour aborder le rôle de la France durant le conflit, chacune permettant de réaffirmer la complexité du contexte franco-irlandais.

Une première piste est offerte à la propagande anti-alliée par le traitement du thème religieux, passé et présent. Dans le contexte difficile qui suit la chute du député irlandais Charles Stewart Parnell en $1890^{32}$, les anti-parnellites, souvent catholiques conservateurs, s'opposent aux parnellites, généralement plus radicaux. La France devient un objet de débat. Les uns l'utilisent comme l'exemple d'une société en décadence morale, les autres prennent sa défense. Le concept d'un peuple resté fidèle aux enseignements du catholicisme, mais prisonnier de ses dirigeants, est alors développé. C'est ce même concept qui est utilisé pour démontrer l'amoralité des responsables français pendant la guerre, opposée à un peuple remplissant bravement son devoir. Une façon de neutraliser les arguments pro-français tout en se gardant de s'attaquer au peuple de France et à ce qu'il représente dans l'histoire irlandaise. Voici comment l'Honesty, qui avait publié quelques jours auparavant le petit article isolé sur l'immoralité française cité plus haut, 
change partiellement de cap. L'athéisme français reste dénoncé, mais l'article vise les responsables politiques de France, alors qu'il rend hommage à son peuple :

\begin{abstract}
Here is this unfortunate country, with the invader in possession of its choicest territory, and a much hated invader he is. The men of France are sacrificing everything that is dear to them in an effort to drive out the detested enemy. And while they are doing this the deposed oligarchy that rules the country is fattening upon its miseries. This shows what kind of patriot an atheist usually is ${ }^{33}$.
\end{abstract}

Pour les nationalistes irlandais qui adhèrent à cette théorie, le peuple français, dont les vertus ne sont pas à remettre en cause, se trouve prisonnier des dirigeants athées et anticléricaux que sont Raymond Poincaré, George Clémenceau, ou encore René Viviani ${ }^{34}$. Le sentiment d'amitié envers la France qui émane de ce texte est encore plus frappant si on l'oppose à la vision assez dure qui est donnée de l'Allemagne. Celle d'un envahisseur en possession de territoires, l'Alsace et la Lorraine, qui ne lui appartiennent pas. Pourtant, dès le début de la guerre, plusieurs Irlandais séparatistes se tournent vers l'Allemagne. Des contacts assez avancés ont existé entre des nationalistes irlandais, notamment sir Roger Casement, et l'état-major allemand. Des armes ont notamment été sur le point d'être envoyées en Irlande. Présenter l'Allemagne comme un "envahisseur " est donc dans ce cadre une démarche tout à fait surprenante. Que faut-il comprendre? L'Allemagne est une pièce maitresse des politiques nationalistes anti-alliés mises en place pendant la guerre, et cette phrase ne permet pas de réévaluer ce sentiment. En revanche, nous touchons ici à la question de l'Alsace-Lorraine, vecteur d'un puissant symbolisme pour une Irlande qui se considère, elle aussi, occupée. En effet, après la défaite de 1870, l'opinion catholique et nationaliste irlandaise a toujours démontré une grande empathie envers les peuples de ces deux régions ${ }^{35}$.

Une autre solution pour résoudre la «question française » consiste simplement à ignorer la France ${ }^{36}$. La Russie, cible facile notamment par sa politique polonaise, est présentée seule à combattre aux côtés des Anglais. Le 11 décembre 1915 l'Hibernian, l'un des rares journaux catholiques et radicaux, accuse ainsi la Russie d'être à l'origine du conflit mondial. Le 24 janvier 1915, dans le Nationality, on peut lire ce commentaire sarcastique :

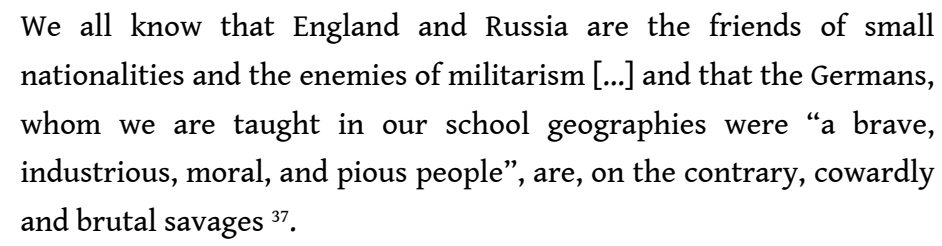

21 L'article évoque ensuite les drapeaux mêlés de Moscou et de la Grande-Bretagne. Rien n'est dit sur le drapeau français, ou sur l'implication française dans la guerre, puisque dans la mémoire et l'identité nationaliste irlandaise, la France a toujours été représentée comme la première alliée de ces petites nations dont parle l'article. Mais la solution la plus communément choisie par les nationalistes radicaux est certainement de présenter la nation française comme une autre victime d'une guerre bâtie, comme Eire-Ireland l'explique, à $95 \%$ par les Anglais ${ }^{38}$. Au contraire de l'Angleterre ou de la Russie, la France n'est donc pas, dans cette perspective, un agresseur. Déjà en 1904, quand la France signe l'Entente Cordiale avec l'Angleterre, Griffith présente l'accord comme le résultat de la séduction des naïfs Français par les Anglais ${ }^{39}$. C'est également ce que l'Irish Freedom, 
journal de l'IRB, affirme dans son numéro d'avril 1914: «La France est écrasée sous l'égoïste monopole anglais ${ }^{40}$. " La guerre est donc une occasion parfaite pour insister sur la machination de la "perfide Albion ». Le sacrifice des soldats français sur les champs de bataille est souvent opposé à l'attentisme anglais. Dans l'Honesty du 4 mars 1916 on peut lire :

The Frenchman knows that since the beginning of the war there are more than 700000 killed, and nearly 800000 permanently disabled, when the British Empire counts hardly 130000 killed $^{41}$.

À la fin de la guerre, la Grande-Bretagne compte approximativement 750000 morts ${ }^{42}$. En conséquence, le chiffre de 130000 morts pour mars 1916 apparaît bien en dessous de la réalité, même s'il est vrai que les états-majors laissent filtrer très peu d'informations sur ce type de statistiques. La volonté de démontrer les manipulations et la lâcheté des dirigeants de Grande-Bretagne est en tout cas claire.

L'article de l'Honesty semble en fait répondre à un double objectif. En effet on y remarque la volonté de présenter le début d'une dissension entre les alliés en insistant sur le terme : "The Frenchman knows ». Les nationalistes irlandais radicaux ont toujours considéré l'entente franco-anglaise comme un accord n'obéissant à aucune logique, et ne pouvant durer comme la discorde ne pourrait manquer de s'imposer entre les deux ennemis héréditaires. Naturellement, alors que la guerre s'installe en Europe, la presse nationaliste radicale insiste sur de supposées tensions entre les soldats français et anglais. Eire-Ireland écrit le 13 novembre 1914 :

There is a rather bitter feeling growing between the French and English soldiers in the field. The French think the English have not "played the game" ${ }^{43}$.

Cette dernière expression insinue que les Français s'éveillent finalement à la réalité et comprennent avec qui leur destinée est liée. L'Entente Cordiale ne pouvait résister, passé le premier combat révélateur des véritables intentions anglaises. En fait, un certain nombre de ces nationalistes radicaux ne semblent pas capables, même après dix années, à se résoudre à une "entente " qui dure toujours. À accepter l'alliance "naïve ", d'une certaine façon la trahison de leur premier et seul allié naturel ${ }^{44}$. Même les plus fervents partisans d'une alliance avec l'Allemagne conservent sur ce sujet un sentiment particulièrement amer. Sir Roger Casement, dans un conte non publié qu'il a écrit luimême, présente ouvertement son opinion:

Once upon a time there were two friends [La France et l'Angleterre naturellement] and they said: "It is a great Pity we are not three for Three's Company Two's none." "Humph!" Said an old woman, "I heard that put differently when I was young." "When you were young, Ma'am", they said with a smile, "people were foolish enough to speak the truth" 45 .

La vieille femme du texte correspond à la représentation traditionnelle de l'Irlande née de la ballade Shan Van Vocht, dont le titre se traduit lui-même par la "vieille dame ", et qui a été composée en souvenir du débarquement des troupes françaises sur les côtes irlandaises en 1798. La référence à la " vieille dame » est celle d'une Irlande soutenue par 
une France révolutionnaire, alors en guerre contre l'Angleterre. Cette vision de la France constitue la "vérité » dont parle le texte, opposée au mensonge d'une alliance francoanglaise. L'Irlande séparatiste, prônant un nationalisme radical, qui pense souvent en terme de religion, de race, de mémoire, ne peut ni admettre, ni même peut-être pleinement comprendre un accord passé entre deux pays n'ayant majoritairement pas la même religion, supposément pas la même origine ethnique (Celtes ou Latins contre Saxons), et partageant une mémoire de conflits et de rivalités.

\section{La question française après Pâques 1916} caractérisée pendant une bonne partie du xix siècle de profondes et durables modifications. La première victime en est le parti parlementaire de John Redmond, qui perd peu à peu le fil des politiques nationalistes. Cette nouvelle situation est illustrée par les changements appliqués à la représentation de la France au sein de la propagande du Sinn Féin. En effet, le front anti-alliés peut maintenant s'appuyer sur une opinion outragée par les exécutions des responsables du soulèvement. Le Sinn Féin bénéficie de cette évolution, et voit sa popularité augmenter sensiblement jusqu'en août $1917^{46}$. Pour le gouvernement français, le progressif retournement du rapport de forces entre nationalistes séparatistes et parlementaires arrive au plus mauvais moment. La répression qui suit le soulèvement de Pâques a pour effet de réduire drastiquement le nombre de recrues irlandaises, alors qu'il faut remplacer les 286000 soldats morts pendant l'année ${ }^{47}$. Différentes tactiques de propagande sont donc employées, mais elles se soldent toutes par un échec ${ }^{48}$.

la propagande anti-alliés, il est temps de se montrer plus agressif. D’autant que si le Freeman's Journal multiplie les articles pro-français ${ }^{49}$, il ne se remettra jamais de la destruction de ses locaux pendant l'insurrection ${ }^{50}$. Aux précautions du début succèdent donc des attaques bien moins teintées de respect pour le lien identitaire et historique franco-irlandais. Le Nationality, chef de file de la propagande anti-alliés à partir de 1916, montre la voie en mettant en valeur l'anticléricalisme français avec de plus en plus d'insistance ${ }^{51}$. Le journal n'hésite pas non plus à retourner contre la France l'argument des provinces perdues, si sensible dans les premières années de guerre, en remettant en cause la légitimité des Français à se saisir de ces territoires : «As to Alsace-Lorraine, the Alsace-Lorrainers are three-fourths German and less than one-fourth French ${ }^{52}$.»

Pour soutenir sa démarche, le Nationality peut compter sur des périodiques qui avaient jusque-là montré un profil neutre, ou légèrement favorable aux alliés. C'est le cas du Catholic Bulletin, un mensuel catholique proche du Sinn Féin. Si en 1914 il soutient la France, ses positions se radicalisent nettement en faveur de l'Allemagne après le soulèvement de 1916 et la crise qui suit les rumeurs d'application de la conscription en Irlande au printemps 1918. En mars de cette même année, il appuie donc la tactique du Nationality sur la question de l'Alsace-Lorraine :

The argument that these two provinces in the main belonged to France before 1870 is not very convincing in supporting the French claim [...] there is another view of the question. What think the Alsatians and Lorrainers now ${ }^{53}$. 

1918, refuse de siéger à Londres, et constitue un parlement à Dublin, le Dáil. En quête de reconnaissance internationale, le mouvement nationaliste irlandais met en place un bureau à Paris, lieu du déroulement de la conférence de la paix. Charles Gavan Duffy notamment met en place un vrai réseau européen de propagande, et déploie d'importants efforts pour ramener à la cause irlandaise la population et le gouvernement français. Mais ce dernier dépend trop de l'Angleterre pour imposer ses vues sur le traité de paix, et assurer sa sécurité face à l'Allemagne. De plus, le soulèvement de Pâques 1916 n'a rien fait pour développer l'amitié et la confiance du gouvernement français envers le mouvement nationaliste irlandais tel qu'il s'impose à partir de 1918-1919.

Mais l'étude de cet effort de propagande nous permet d'observer qu'au-delà de la nette baisse d'influence de la France sur les politiques nationalistes à partir de 1904, sa représentation, associée aux épisodes de 1798 ou 1848, oblige, pendant la Première Guerre mondiale, les héritiers de ces événements à s'accommoder de sa présence au sein de l'identité nationale irlandaise jusqu'à ce que l'état de l'opinion publique irlandaise change drastiquement après le soulèvement de Pâques 1916. Plutôt que de le renier, il semble plus logique d'accepter le lien de deux nations dont les identités se sont inter-influencées, et de n'en rejeter que les dirigeants, britanniques ou français anticléricaux, qui les gardent prisonnières. Ceci nous permet également de garder en mémoire l'importance du contexte continental en Irlande, qui, s'il a été porteur d'espoirs déçus au cours du XIX siècle, n'en a pas moins continué à influencer les politiques nationalistes irlandaises jusqu'à la veille de la signature du traité anglo-irlandais de 1921. 


\section{NOTES}

1. Keith Jeffery, Ireland and the Great War, Cambridge, Cambridge University Press, 2000, p. 1. Aux publications largement utilisées dans cet article, nous pouvons aussi rajouter des études d'histoire militaire comme celle de Timothy Bowman, Irish Regiments in the Great War (Manchester, Manchester University Press, 2003), des études régionales et personnelles telle Niall McGinley, Donegal, Ireland and the First World War (Letterkenny, An Crann, 2005) ou encore l'ouvrage de T. P. Dooley, Irishmen or English Soldiers (Liverpool, Liverpool University Press, 1995).

2. Soulèvement planifié par l'organisation séparatiste de l'Irish Republican Brotherhood. La répression qui a suivi l'opération a été à l'origine de la montée en puissance du Sinn Féin au sein de l'opinion nationaliste irlandaise. Griffith n'a pas participé aux opérations militaires, mais a su en tirer profit en dénonçant la violence des autorités britanniques.

3. Cela fait une vingtaine d'années que des recherches sont menées sur ce sujet. Parmi les publications récentes, on peut noter le livre de John Horne (dir.), Our War (Dublin, Gill\&MacMillan, 2008). L'ouvrage, qui traite notamment des vétérans, montre un intérêt grandissant de la recherche et du public pour l'histoire des soldats irlandais pendant et après la Grande Guerre.

4. Keith Jeffery, Ireland and the Great War, op. cit., p. 6.

5. . Jérôme aan de Wiel, The Irish Factor 1899-1919, Dublin, Irish Academic Press, 2008, p. 161.

6. Les recherches effectuées sur le sujet sont nombreuses, mais on peut citer entre autres les travaux de thèse de Janick Julienne, La question irlandaise en France de 1860 à 1890 : perceptions et réactions (thèse de doctorat, 1997, Université Paris 7), et de Laurent Colantonio, Daniel O'Connell: un Irlandais au coeur du débat politique français, (thèse de doctorat, 2001, Université Paris 8), l'article de Vincent Comerford sur l'influence française du mouvement fenian, «France, fenianism, and Irish nationalist strategy " ( Études Irlandaises, $\mathrm{n}^{\circ} 7,1982$ ), ou bien encore, sur l'Irlande et la guerre franco-prussienne de 1870, la publication de Gary K. Pealing, "Saxon and Celt on the Rhine? Religion and representation in Irish reactions to the Franco-Prussian war 1870-71», in Colin Graham, Leon Litvack (dir.), Ireland and Europe in the Nineteenth Century (Dublin, Four Court Press, 2005), pp. 112-121; ainsi que sur le mouvement Jeune Irlande, les articles de Pierre Joannon, «L'Irlande et la France en 1848 » (Études Irlandaises, $N^{\circ} 12,1987$ ) et de Mary Buckley, « French influences on Young Ireland, 1842-45 » (Études Irlandaises, $N^{\circ} 7,1982$ ).

7. On pense notamment aux brigades irlandaises au service des armées de Louis XIV ou de Louis $\mathrm{XV}$, à l'intervention militaire française durant la rébellion de 1798 menée par l'organisation nationaliste des Irlandais Unis, ou à l'influence de la Révolution de Février sur le mouvement nationaliste romantique des Jeunes Irlandais. Dans les années 1850-1860, on peut aussi noter les activités à Paris de nombreux membres de l'organisation de l'Irish Republican Brotherhood, appelés fenians.

8. C'est ce qu'explique Jérôme aan de Wiel dans The Catholic Church in Ireland 1914-1918, Dublin, Irish Academic Press, 2003, p. 128. 
9. Ibid., p. 129.

10. Ibid., p. 135.

11. Ibid., p. 222. On compte tout de même un nombre non négligeable de 95143 recrues irlandaises engagées dans l'armée britannique entre août 1914 et février 1916.

12. Jérôme aan de Wiel, The Irish Factor..., op. cit., p. 181.

13. Fraternité irlando-américaine établie aux États-Unis en 1836, d'inspiration catholique et nationaliste. Avec l'arrivée de Joseph Devlin en 1905, elle se transforme en véritable machine politique au service du parti parlementaire irlandais de John Redmond. Jusqu'alors peu influente en Irlande et en Grande-Bretagne, elle va très largement se développer au sein de la communauté catholique, notamment en Ulster.

14. Jérôme aan de Wiel, The Catholic Church..., op. cit., p. 136.

15. Weekly Freeman, 19 septembre 1914.

16. Discours compilés dans La délégation irlandaise à Paris 1915, Paris, Printing Chaix, 1915.

17. Weekly Freeman, 26 septembre 1914.

18. Honesty, 15 janvier 1916.

19. Jean-Baptiste Duroselle, La France de la "belle époque ", Paris, Presse de la Fondation Nationale des Sciences Politiques, 1992 (2 ${ }^{\text {nde }}$ éd.), p. 12.

20. Nationality, 24 juillet 1915. Griffith étant connu pour son racisme chauvin, il est possible qu'une telle déclaration tienne autant de la propagande que de ses convictions personnelles.

21. L'article cité ci-dessus est à la fois le premier et le dernier du genre à être publié dans l'Honesty.

22. Jérôme aan de Wiel, The Irish Factor..., op. cit., p. 159. Rapport qui s'intéresse au comté de Roscommon.

23. On pense notamment au Freeman's Journal, à l'Irish Independent, ou au Cork Examiner, qui sont parmi les journaux les plus lus du pays.

24. Dans sa thèse de doctorat, Janick Julienne montre très bien la façon dont le gouvernement français pratique une politique de moins en moins amicale envers les nationalistes irlandais à partir des années 1870. L'échec relatif de la visite de Parnell en 1881 puis l'expulsion de James Stephens en 1885 en font la démonstration. Cependant, il nous semble que cet argument peut être complété par une analyse plus poussée d'archives mettant en valeur les réactions irlandaises à ces politiques françaises.

25. Patrick Maume, The Long Gestation, Irish Nationalist Life 1891-1918, Dublin, Gill \& Macmillan, 1999, p. 54.

26. Pour les années 1880 , on pense notamment à l'Irishman dont l'un des principaux responsables, James O'Connor, est un membre influent de l'IRB, ou, dans les années 1890, au Weekly Irish Independent, qui compte dans sa rédaction plusieurs membres de l'organisation séparatiste, ainsi qu'au United Irishman d'Arthur Griffith, qui participe à la renaissance du séparatisme irlandais.

27. La neutralité de la France pendant la guerre des Boers est défendue par Pascal Venier, dans «French Foreign Policy and the Boer War », in Keith Wilson (dir.), The International Impact of the Boer War, (Chesham, Acumen, 2001), p. 65-78. Mais dans The Irish Factor..., op. cit., p. 6, Jérôme aan de Wiel défend la thèse selon laquelle certains diplomates français, notamment Jules Cambon, ont très vite compris l'importance du « monde irlandais ». 
28. The Gael, 29 janvier 1916. Le Gael reste un journal qui s'appuie beaucoup sur une propagande très pro-allemande, et cette caractéristique rend donc cet article d'autant plus intéressant.

29. Dans The Irish Factor..., op. cit., p. 22-24 et p. 28, Jérôme aan de Wiel expose la détérioration des relations franco-irlandaises après la signature de l'Entente Cordiale. Il montre comment les Irlandais nationalistes se rendent rapidement compte du changement d'attitude du gouvernement français à leur égard, qui voit désormais l'Irlande comme une source possible d'affaiblissement pour l'Angleterre. Ce changement permet à l'Allemagne de gagner du terrain, surtout auprès des Irlandais séparatistes, notamment pendant la crise du « Home Rule » de 1913-1914, où les nationalistes sont à la recherche d'armes en cas de guerre civile avec les unionistes d'Ulster.

30. Tom Garvin, Nationalist Revolutionaries in Ireland, Dublin, Gill and Macmillan, 2005 ( $1^{\text {re }}$ éd. 1987), p. 108-110.

31. Comme on a pu l'observer, le Nationality d'Arthur Griffith hésite moins que d'autres journaux à s'attaquer à la France, à son gouvernement et à sa moralité. Le Spark, proche de l'IRB, est, quant à lui, le seul journal qui ne montre aucune sympathie envers une France qu'il considère moralement inférieure à l'Irlande.

32. Charles Stewart Parnell a dominé la vie politique irlandaise pendant les années 1880. Il meurt en 1891, son honneur souillé par une relation adultérine, et laisse une Irlande nationaliste profondément divisée.

33. Honesty, 22 janvier 1916. Sur le même thème: Hibernian, 4 mars 1916; Nationality, 21 août 1915 et 4 décembre 1915, article qui se concentre sur la figure de Viviani.

34. Jérôme aan de Wiel, The Irish Factor..., op. cit., p. 182. Bien avant la guerre, Viviani est accusé d'être le plus grand anticlérical de France, de surcroît franc-maçon. La dénonciation $\mathrm{du}$ "Grand Orient », complotant à la tête de l'État français, est une autre façon de dissocier peuple et dirigeants.

35. Des journaux nationalistes aux positions politiques aussi différentes que le républicain Flag of Ireland, ou le catholique conservateur Dublin Evening Post, montrent tous les deux une sympathie profonde envers les populations d'Alsace et de Lorraine jusqu'à la fin du XIX $x^{e}$ siècle.

36. C'est aussi la tactique adoptée face aux États-Unis lorsque ces derniers entrent en guerre aux côtés de la Grande-Bretagne en avril 1917. Voir Ben Novick, Conceiving Revolution..., op. cit., p. 114.

37. Nationality, 24 janvier 1915.

38. Eire-Ireland, 2 décembre 1914. Scissors and Paste, un autre journal de Griffith, développe aussi longuement la théorie selon laquelle l'Angleterre est la seule responsable du conflit.

39. Patrick Maume, The Long Gestation..., op. cit., p. 86.

40. Irish Freedom, avril 1914. Le 15 septembre 1915, le catholique Hibernian reprend une thèse similaire en affirmant que l'affaire Dreyfus n'avait été qu'une machination anglaise pour affaiblir la « Gallant French army ».

41. Honesty, 4 mars 1916. Autre article sur le même thème : Nationality, 12 février 1916.

42. Martin Gilbert, First World War, Londres, Harper Collins, 1994, p. 541.

43. Eire-Ireland, 13 novembre 1914. Autres articles sur le même thème: Honesty, 25 mars 1916 ; Nationality, 12 février 1916. 
44. Un sentiment ressenti par le fenian O'Donovan Rossa, alors encore un enfant, lorsqu'en 1848 Lamartine refuse d'apporter son soutien au mouvement de la Jeune Irlande. Voir Jérôme Aan de Wiel, The Irish Factor, op. cit., p. 401.

45. National Library of Ireland, Ms 29064, "The Three Friends». Malheureusement, la rédaction de ce conte n'a pas été datée par l'auteur, mais l'on peut penser qu'il a été écrit avant la guerre, ou pendant ses premiers mois.

46. Jérôme aan de Wiel, The Irish Factor..., op. cit., p. 311.

47. Ibid., p. 294.

48. Une mission ecclésiastique est notamment envoyée en Irlande. Pour une analyse détaillée, voir Jérôme aan de Wiel, The Catholic Church..., op. cit., p. 128-52.

49. Jérôme aan de Wiel, The Irish Factor, op. cit., p. 240.

50. Patrick Maume, The Long Gestation, op. cit., p. 186.

51. Ibid., p. 313.

52. Nationality, 7 avril 1917.

53. The Catholic Bulletin, mars 1918.

54. C'est la thèse développée par David FitzPatrick dans The logic of collective sacrifice: Ireland and the British Army, 1914-1918, The Historical Journal, Vol. 38, n 4, décembre 1995, 1017-1030.

\section{RÉSUMÉS}

Cet article explore la façon dont la presse nationaliste irlandaise a utilisé l'image de la France durant la première guerre mondiale. La radicalisation des politiques nationalistes, entre constitutionnalistes poussant au recrutement et radicaux s'y opposant, est forte dès 1914, encore plus accentuée après Pâques 1916. Combattant aux côtés de la Grande-Bretagne, la France, qui continue malgré tout à bénéficier d'une place à part au sein de l'identité nationale irlandaise, ajoute à la complexité de la situation jusqu'en 1916, car la situation évolue par la suite. C'est ce rôle unique que nous chercherons à comprendre. Entre utilisation des symboles du traditionnel lien franco-irlandais pour les uns, et rejet de cet héritage pour ceux qui l'avaient longtemps mis en valeur.

This article explores how the Irish nationalist press used the image of France during the First World War to feed its own divisions. The radicalization of nationalist politics, between constitutionalists supporting recruitment, and radicals opposing it, is pronounced from 1914, and even more so after Easter 1916. France, which fought alongside great Britain, still enjoyed a special place in Irish nationalism. This relationship became more complex after 1916 and the representation of France changed as a result of the Easter Rising. The present article seeks to explore this representation, caught between the traditional symbols of Franco-Irish complicity and the rejection of this heritage by certain sectors of the community which had so long cherished it. 
INDEX

Keywords : First World War, Franco-Irish relations, press, history of representations, Irish nationalism

Mots-clés : relations franco-irlandaises, nationalisme irlandais, Première Guerre mondiale, presse, histoire des représentations

\section{AUTEUR}

\section{PIERRE RANGER}

Université de Paris XII - Val de MarneQueen's University, Belfast 